\title{
Development of a 3D-printed device evaluating the aerodynamic performance of rotary wings
}

\author{
Keiichiro TAKATO* and Susumu SHIRAYAMA** \\ ${ }^{*}$ Nishinippon Institute of Technology \\ 1-11 Aratsu, Kanda-machi, Fukuoka 800-0394, Japan \\ E-mail: takato@nishitech.ac.jp \\ ${ }^{* *}$ Department of Systems Innovation, School of Engineering, the University of Tokyo \\ 7-3-1 Hongo, Bunkyo-ku, Tokyo 113-8656, Japan
}

Received: 30 October 2017; Revised: 12 January 2018; Accepted: 18 February 2018

\begin{abstract}
Applications for remotely operated rotary wing aerial vehicles, hereafter referred to as drones, are rapidly expanding in scope and such aircraft may soon become an integral part of everyday life as well as a source of innovation. However, it is quite difficult to improve drone flight performance and reduce development costs because performance optimization depends strongly on the intended application. As a result, drones are currently developed by trial and error using various rotary wing shapes. In this study, a device for evaluating the aerodynamic performance of rotary wings is proposed. We consider it likely that our proposed device will be useful for various drone-related applications in addition to facilitating drone development because it is inexpensive to produce and can eliminate the need for large expensive laboratory equipment such as wind tunnels when performing flight characteristic evaluations. In this paper, we report on the fabrication of our 3D general fused deposition modeling (FDM) printed device along with some experimental results.
\end{abstract}

Keywords : FDM 3D printer, Rotary wing, Aerodynamic performance, Taketombo, Drone

\section{Introduction}

Remotely operated rotary wing aerial vehicles, hereafter referred to as drones, are currently being used for a variety of purposes such as geographical surveys, disaster prevention surveillance, and large equipment inspections, as well as logistic deliveries to isolated points. Furthermore, the use of such devices is expanding to include infrastructure inspections and other roles (Nonami 2017; Keeney 2015). However, various difficulties arise when researchers attempt to improve drone flight characteristics and restrain development costs.

One reason is the difficulty of repurposing a drone that was developed for a specific function to another role. In other words, a general-purpose design method for drones has not yet been established. Another reason is the difficulty in acquiring information needed for aerodynamic performance evaluations of rotary wings. For example, it is difficult to estimate the lift and drag acting on a rotary wing with different Reynolds numbers or/and different airfoil sections. This is because rotor quality assessments require large experimental devices and the associated measurement processes require the services of persons skilled in operating such equipment.

For these reasons, drone airframes are currently manufactured by trial and error using various rotary wing shapes (Brandt and Selig 2011; Shetty and Selig 2011), which increases development costs and becomes a factor preventing the expansion of drones into other practical uses.

In this paper, we report on the development of a device that is capable of both improving drone performance and reducing related development costs. Our proposed device makes it possible to evaluate the flight characteristics of a wide variety of rotary wings used in drones while eliminating the need for testing in large experiment equipment such as wind tunnels. For instance, easy evaluation of the aerodynamic forces on the rotary wing makes the process of searching a rotary wing configuration with a high lift to drag ratio more efficient. Furthermore, fabrication of our device is inexpensive and easy due to the use of $3 \mathrm{D}$ general fused deposition modeling (FDM) printing. In the sections 
below, we will report on the development of our device along with some experimental results obtained.

\section{Conceptual design}

\subsection{Inspired by a flying toy taketombo}

Previously, rotary wing aerodynamic performance evaluations have almost always been performed using wind tunnels or similar systems. However, wind tunnel experiments are influenced by the method used to present the rotor being tested, and the reproduction of flight states, as well as the transmission and acquisition of sensor/control data required for drone operation, is difficult when an actual drone is used.

Additionally, since rotor/body interference presents its own problems, it is important to evaluate the performance of a rotor under standalone conditions. Considering these issues, the following three items were established as requirements for the design of aerodynamic ability evaluation equipment.

(I) It must be possible to grasp the flow around the rotor blade in various flight conditions.

(II) It must provide repeatability for specific flight conditions.

(III) It must have fewer restrictions on flight location, and be inexpensive to produce and easy to use.

The requirements of Items (I) and (II) are those for handling various flight conditions. However, if various flight conditions are handled, the requirements in Item (III) are difficult to satisfy, for the reasons already described above.

Therefore, we began our approach to the problem by examining a flying toy taketombo, which are popular toy helicopters commonly made from bamboo that consist of rotor blades attached to a simple shaft that forms a vertical axis (for more details, see Ref. (Takato and Shirayama 2012)). However, it is difficult to satisfy the requirements in Item (II) using a human-launched taketombo model because air patterns and flight forms are difficult to reproduce when the device is launched via human hands (Yasuda 2008; Yasuda 2016).

For example, in a related study, Yasuda conducted flight experiments using taketombo launcher (Yasuda 2016). Reproducibility of the flight path of the taketombo is ensured by the launcher proposed in Ref.(Yasuda 2016), but it will be considered that a number of flight conditions could not be achieved with such a simple device. Additionally, since the launcher in Ref. (Yasuda 2016) does not meet the requirements of Item (III), and considering our requirement for a design that also satisfies Item (II), we decided to develop a new launcher that is based primarily on preceding research(Takato and Shirayama 2012). However, it is difficult to confirm whether the requirements of Item (II) were met in this study. Furthermore, the requirements for a design that satisfies the restrictions imposed by Item (III) are difficult to achieve for aerodynamic performance evaluation equipment due to the need to utilize generally available parts, ensure equipment portability and ease of installation (choosing the installation location), ensure safety, and consider environmental factors. Moreover, the flight envelope of the taketombo itself becomes a problem.

In order to solve these problems, taketombo takeoffs from human hands (hereafter referred to as hand-launched takeoffs) will be analyzed anew. An example of a typical hand-launched taketombo flying perpendicular to the takeoff point is shown in Fig. 1

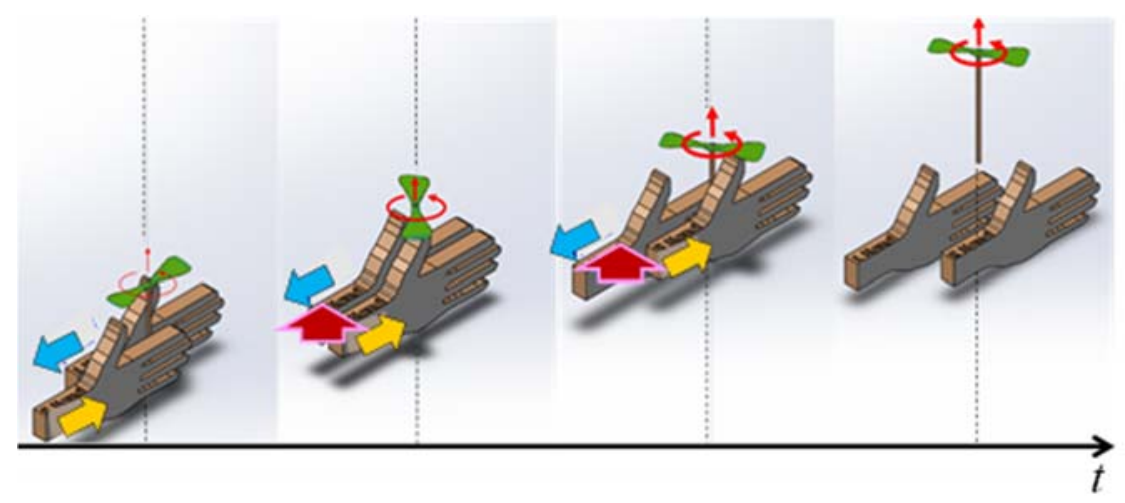

(i)

(ii)

(iii)

(iv)

Fig. 1 Schematic diagram of a hand-launching. (i) Grip and rotation start, (ii) Rotation acceleration, (iii) Maximum rotation speed, (iv) Launch. 
Axial shaft rotation is produced by the counter movements of the user's two palms, and lift generated is by the rotation of the rotary wing. The generated lift causes the taketombo to take flight after the user's palms have separated from the axial shaft. In addition, the operator's arms are often used to add a catapult action to the rotation-induced lift produced by his/her hands, and hand-tilt off the vertical direction can add a horizontal vector to the taketombo flight. Although the basics of this flight method are simple, a variety of factors, including the axial rotation method and axial direction, must be considered when attempting high altitude and long-distance flights.

Accordingly, in order to elucidate a conceptual design for a hand-launched takeoff, we began by classifying the operation into four states, as shown below:

(i) Grasping and rotation start: The axial shaft of taketombo is gripped and rotated. The axial component (shaft) remains gripped between the user's palms.

(ii) Rotation acceleration: The rotational speed of the taketombo is increased as the user presses the shaft between his/her two palms and moves his/her hands in opposite directions.

(iii) Maximum rotation speed: As shown in the figure, rotation acceleration is completed when the axial shaft begins to separate from the bottom palm of one hand and the fingertips of the opposite hand.

(iv) Release: The axial shaft is fully released. (Sometimes release may occur at the end of (iii) above.)

Next, it was necessary to create a mechanism for mimicking a hand-launched takeoff, in addition to adding a catapult action to items (i) to (iv). Consideration must also be given to cases of forward or backward movement. The result is five required mechanisms: axial shaft grasping, axial shaft rotation, axial shaft release, axial shaft inclination, and catapult action. In this paper, imitation of catapult action and axial inclination were set aside for future study and attention was focused on the other three mechanisms.

\subsection{Grasp and rotation}

The imitation of hand-launched axial grasping and axial rotation was attempted first.

As shown in Fig. 1, taketombo axial shaft grasping is carried out by placing the shaft between the user's palms, and rotation is imparted by palm movements. Fig. 2(a) shows a simplified breakdown of this action. In this figure, the shaft (S) is set between two plates shown by (A) and (B), and then rotated by steady opposing movements of the two plates. Here, the upward movement of the right plate and downward movement of the left plate causes the shaft to rotate in a counterclockwise direction. In order to impart sufficient rotation via this mechanism, high-speed devices such as caterpillar drives or belt sanders would be needed, thus making it difficult to fulfill Item (III) requirements.

The method by which the circular objects of A, B, and C in Fig. 2(b) are pressed against the axial shaft (S) to impart rotation is hereafter referred to as the drive circle. In this method, a minimum of three drive components are needed to produce a stably rotating axial shaft.

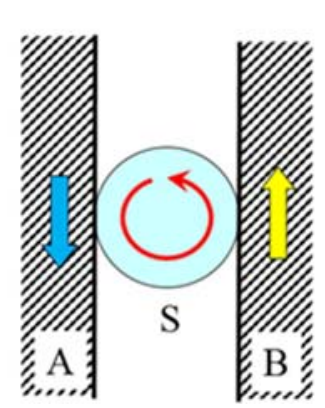

(a) Two moving flat plates

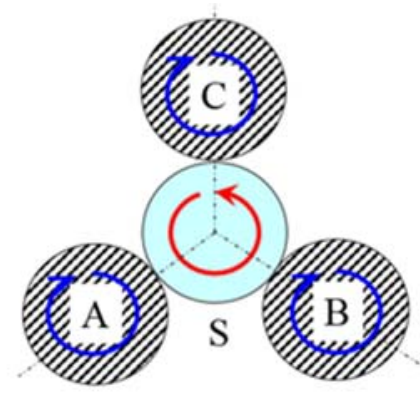

(b) Three rotating disks

Fig. 2 Conceptual diagram of gripping and rotating a shaft

Fig. 2(b) show the fundamental axial shaft grasping and rotating mechanism. On the other hand, when rotating the axial shaft of a taketombo, the ratio of the diameter of a driven shaft to the diameter of a drive circle must be taken into 
consideration. In order to stably grasp the driven axial shaft and to transmit sufficient torque to the shaft for inducing the rotation speed, it is necessary to increase the diameter of the drive circle in comparison with the driven shaft.

More specifically, when grasping with three drive circles of the same diameter $D, D$ becomes $D=3 d /(2 \sqrt{3}-3)$ if the diameter of a driven shaft is set to $d$. This formula about $D$ can be obtained using the condition that three drive circles on the same plane contact each other and the driven shaft contact to the three drive circles. In the case of four drive circles, $D=d /(\sqrt{2}-1)$. Thus, the size of the determined drive circle restrains the design. Note that drive circles exist in three dimensions, as seen in the conceptual diagram shown in Fig. 3.

In this figure, we can see how it becomes possible to grasp and rotate the axial shaft of a taketombo using three drive circles. However, since the stiffness of a taketombo's bamboo axes is low, if excessive grasping power is used, the axial shaft will be bent, as seen in the dashed line (S') of Fig. 3(b). Accordingly, it is appropriate to set two drive circles to serve as a pair so that they can grasp and rotate the axial shaft as one set. Fig. 4 shows two drive circle sets arranged in 3-dimensional space, which efficiently allow the driven axial shaft to be grasped and rotated. The figure also shows that distortions to the low rigid axial shaft are suppressed because the two drive circle sets (labeled $\alpha 1, \alpha 2$ and $\beta 1, \beta 2$ ) face each other.

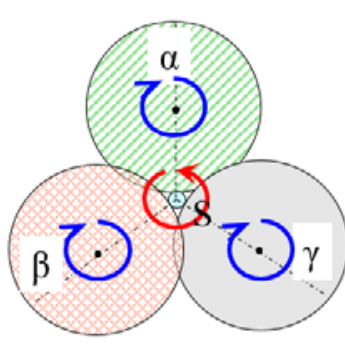

(a) Top view

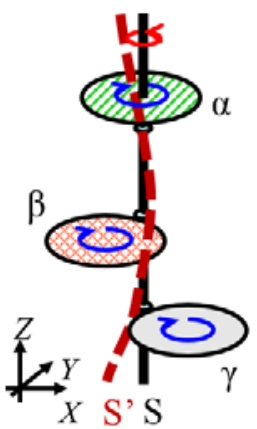

(b) Isometric view

Fig. 3 Gripping and rotating shaft with three disks arranged in 3-dimensional space

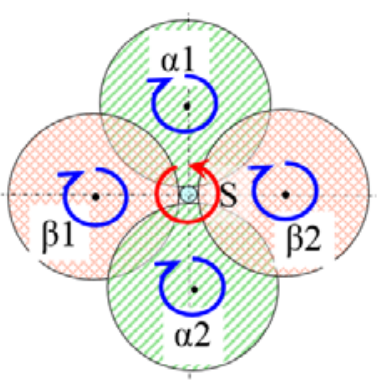

(a) Top view

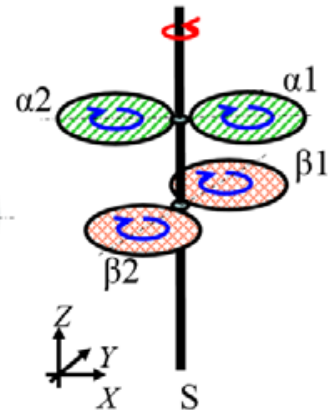

(b) Isometric view
Fig. 4 Shaft grasping by four driving circles arranged in 3-dimensional space

\subsection{Conceptual construction of an open mechanism 2.3.1 Proposal A}

In addition to grasping the shaft and rotating the shaft as discussed in section 2.2 , an opening action is required due to the separation of the driving circles from the driven shaft, as described in operation state (iii) of section 2.1. The conceptual design for achieving mechanical takeoff of this research consists of three mechanisms, axial shaft grasping, axial shaft rotation, and axial shaft release. In the field including industrial robots, objective research into grasping and release has been conducted over the course of numerous years (Chen 1982; Lee 1988). In addition, there have also been many rotation-only mechanisms reported in this field. However, few studies combine continuous movements including gripping, rotation, and release. Of course, taketombo launchers, which have been little studied, have had no research on these continuous movements.

For example, in Tsuneya et al. (Tsuneya and Tsutsumi 1992), the driving motor axis and driven taketombo axial shaft are arranged and connected in a straight line, while in Yasuda(Yasuda 2016), in which grasping was imitated, the rotation was produced by a falling weight. In both studies, it was thought that launchers would not be designed to imitate human actions. Moreover, the stability and repeatability of action provided by the open mechanism of the driving and driven shafts resulted in problems.

For these reasons, as shown in Fig.5, a method of separating the four drive circles from the bamboo axial shaft at the shortest straight line distance was studied. In addition, based on the method shown in Fig.6, a prototype launcher was fabricated (Takato and Shirayama 2012; Takato and Shirayama 2013). In this method, each of the four drive circles is equipped with a drive motor and is supported by a large spring. When support provided to the spring is released, a drive circle is separated from a driven shaft, and the axial shaft is released.

When the experimental launcher fabricated in preceding research (Takato and Shirayama 2012; Takato and 
Shirayama 2013) is used, a taketombo will repeatedly fly in the same manner if the same conditions are used, thereby allowing a flight form to be confirmed. However, this result is dependent on various conditions, such as adequately fixing the mechanism to a heavy platform and ensuring sufficiently high-speed rotation to generate lift. Additionally, the characteristics that govern four motor-driven drive circles results in weight increases due to the fixed launching stand requirement, which also limits portability. Furthermore, since all four motors operate independently, synchronous control is required for each motor. Taken together, these factors make it difficult to produce a launcher that is sufficiently small and inexpensive, which means Proposal A cannot fulfill the Item (III) design requirements.

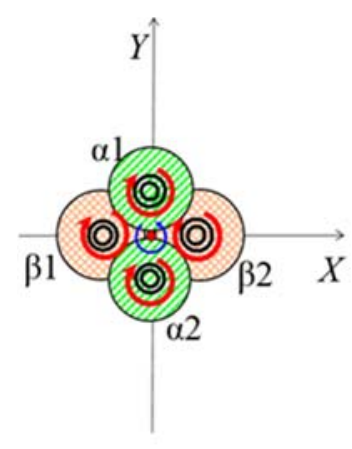

(a) Gripping state

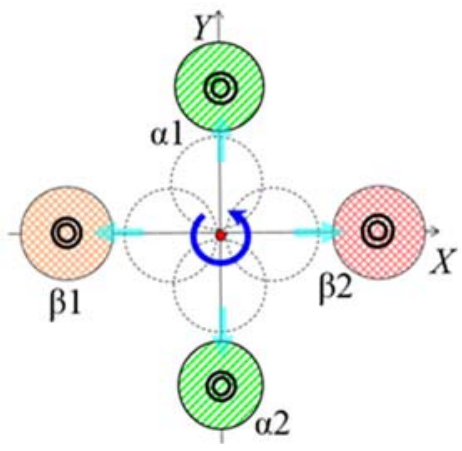

(b) State after release

Fig. 5 Schema of axial shaft release mechanism using linear movements of four disks

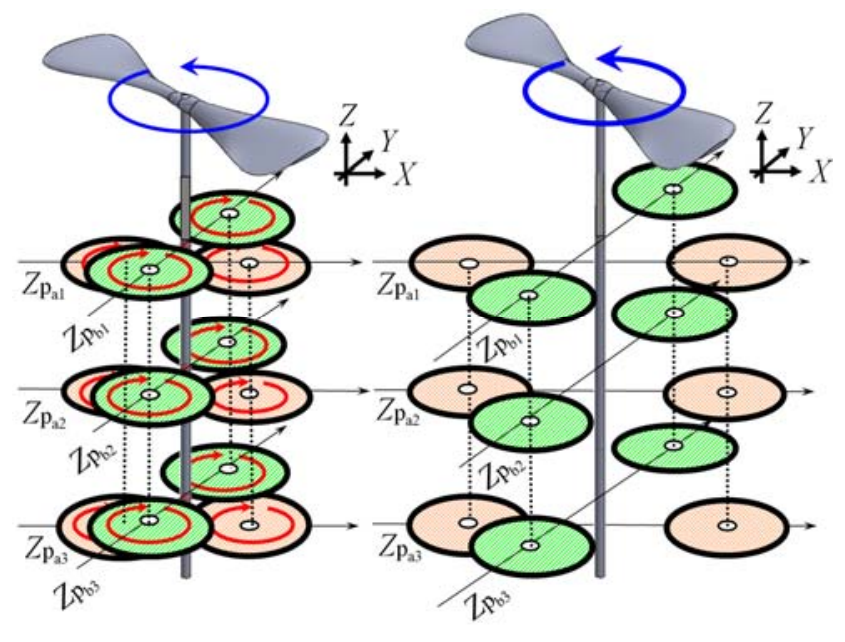

(a) Gripping state

(b) State after release

Fig. 6 Schema of launching mechanism in Proposal A

\subsubsection{Proposal B}

In this section, ideas for miniaturization and weight reduction are described. The design requirements for Items (I) and (III) necessitate quality assessment experiments, which include low altitude flights where the objectives are to travel a short distance and arrive on target.

In such cases, a sufficient number of experiments must be carried out to permit statistical analysis. Therefore, in order to satisfy Item (III), a design must ensure experimental implementation ease.

In the case of a device-launched flying object such as a taketombo, since the flight path and flight form are both determined at launch time, it is necessary to create different performance trajectories that allow the same object to reach the same target using the same launcher control. When weight conservation and device miniaturization are emphasized in a device based on Proposal A, the large mass of the four motors can result in reactions at the time a mounted motor disengages from the axial shaft, which may vibrate the launcher, and thus affect the launch position. As a result, it becomes theoretically difficult for a Proposal A design to meet Item (II) requirements. 
With the above points in mind, this paper focuses on the open mechanism of Proposal B, as shown in Fig. 7. Fig. 7 (a) shows a state in which the axial shaft is gripped by the drive circle. The drive circle made up of $\alpha$-and $\beta$-axes is connected with a newly set-up $\gamma$-axis. Next, the open status is shown in Fig. 7(b). Here, the position of the $\gamma$-axis is fixed and the drive circles of the $\alpha$-and $\beta$-axes rotate in a way that centers on the $\gamma$-axis.

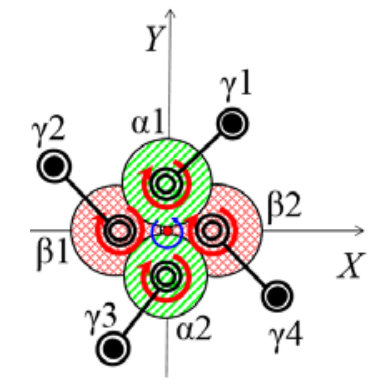

(a) Gripping state

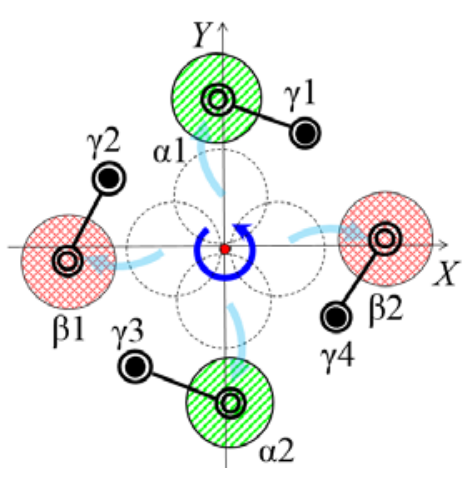

(b) State after release

Fig. 7 Shaft release mechanism based on rotational motion of four disks

Since the distance from the $\gamma$-axis to the drive circle created by the $\alpha$-and $\beta$-axes does not change for this Proposal B model, power transfer can be attained by placing a pulley, gear, or other mechanism between the two axes. As a result, the fixed position of the $\gamma$-axis provides a power source that makes it unnecessary to drive the $\alpha$ - and $\beta$-axes using a motor, as is called for in Proposal A. This means that, compared with Proposal A, reactions are reduced and vibrations, which were the primary cause of launching errors, can be minimized. Moreover, the synchronous control required for four independent power sources called for in Proposal A are also unnecessary.

After selecting Proposal B to handle the axial shaft grasping, axial shaft rotation, and axial shaft release problems, we then tackled launcher miniaturization and number-of-rotations synchronization.

An overview of a typical launcher is shown in Fig. 8. One motor, shown by $(\mathrm{M})$ in the lowermost part of the Fig. 8, provides a power source that drives four $\gamma$-axes via gears (or pulleys). The three drive circles of the $\alpha$ - and $\beta$-axes transfer torque from the purple $\gamma$-axis gears, causing it to rotate while grasping the axial shaft of the taketombo. The four $\alpha$ - and $\beta$-axes move in a circular arc around the fixed in position $\gamma$-axis while rotating, and then release the taketombo axial shaft.

\section{Basic design and detail design 3.1 Design of power transfer mechanism and transfer part}

First, we examined gears based on Proposal B discussed in subsection 2.3.2.

Power is transferred from M, shown in the lower part of Fig. 8, to the axial shaft of the taketombo, shown in the upper part of the same figure. Although, as explained above, the launcher structure of this paper can utilize either a pulley or gear, it was thought that gears were more advantageous in terms of miniaturization, so that usage will be examined hereafter. Nevertheless, it should be noted that the same function is achievable if the effective gear diameter is transposed to the pitch diameter of a pulley.

A breakdown of the $\gamma$-axis shown in Fig. 8 is provided in Fig. 9. The gear connected to $M$ is labeled $\mathrm{GE}_{\mathrm{M}}$, while the gear in the lower part of the $\gamma$-axis (which engages with $\mathrm{GE}_{\mathrm{M}}$ ) is labeled $\mathrm{GE}_{1}$. The gear connected to the upper part of the $\gamma$-axis is labeled $\mathrm{GE}_{2}$, while the gear connected to the $\alpha$-axis and lower part of the $\beta$-axis is labeled $\mathrm{GE}_{3}$. A tire is used to provide a friction equivalent to a pair of human palms in contact with the taketombo axial shaft and is labeled $\mathrm{TI}_{\mathrm{p}}$. As for the size of the gear or tire, the reference diameter of the $\mathrm{GE}_{\mathrm{M}}$ motor gear is $D_{\mathrm{M}}$ and the reference circle of the $\mathrm{GE}_{1}$ gear is $\mathrm{D}_{1}$. The standard semi-circle diameter of the $\mathrm{GE}_{2}$ gear is $D_{2}$ and the reference diameter of the $\mathrm{GE}_{3}$ gear is $D_{3}$. The tire diameter is set to $D_{\mathrm{p}}$ and the axial diameter of the taketombo is set to $d_{\mathrm{b}}$. The motor rotation speed is set at $N_{\mathrm{M}}$. If the moderating ratio of the motor slowdown gear is set to $R_{\mathrm{M}}$, then rotation speed $N_{\mathrm{b}}$ of a taketombo is shown as $N_{\mathrm{b}}=N_{\mathrm{M}} R_{\mathrm{M}}\left(D_{\mathrm{M}} / D_{1}\right)\left(D_{\mathrm{p}} / d_{\mathrm{b}}\right)\left(D_{2} / D_{3}\right)$. Moreover, when the running torque of a motor is set to $T_{\mathrm{M}}$, Torque $T_{\mathrm{b}}$, which drives the taketombo, is expressed as $T_{\mathrm{b}}=T_{\mathrm{M}}\left(N_{\mathrm{M}} / N_{\mathrm{b}}\right)$. In this case, the final transmission ratio from the motor to the taketombo axial shaft serves as $N_{\mathrm{M}} / N_{\mathrm{b}}$. 


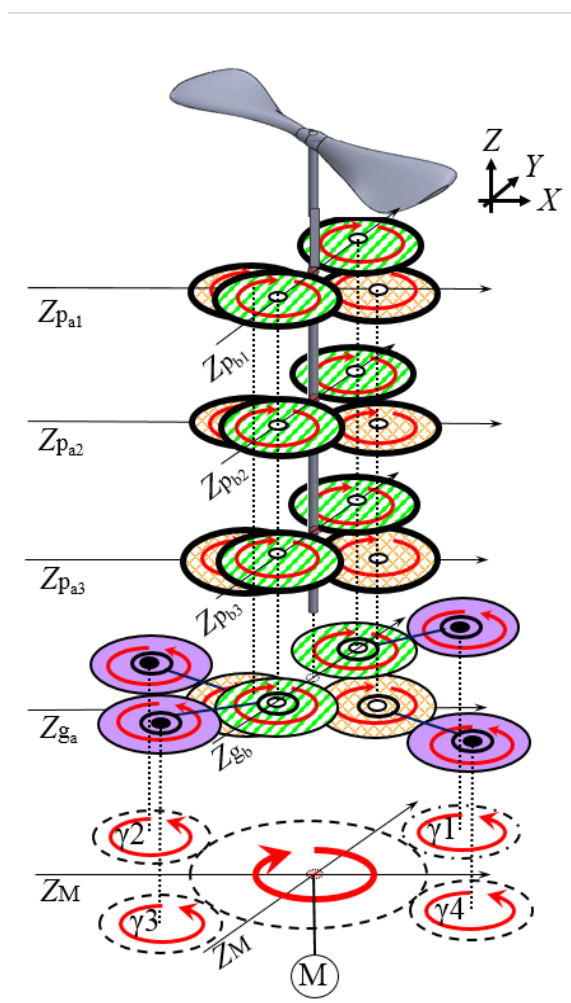

(a) Gripping state

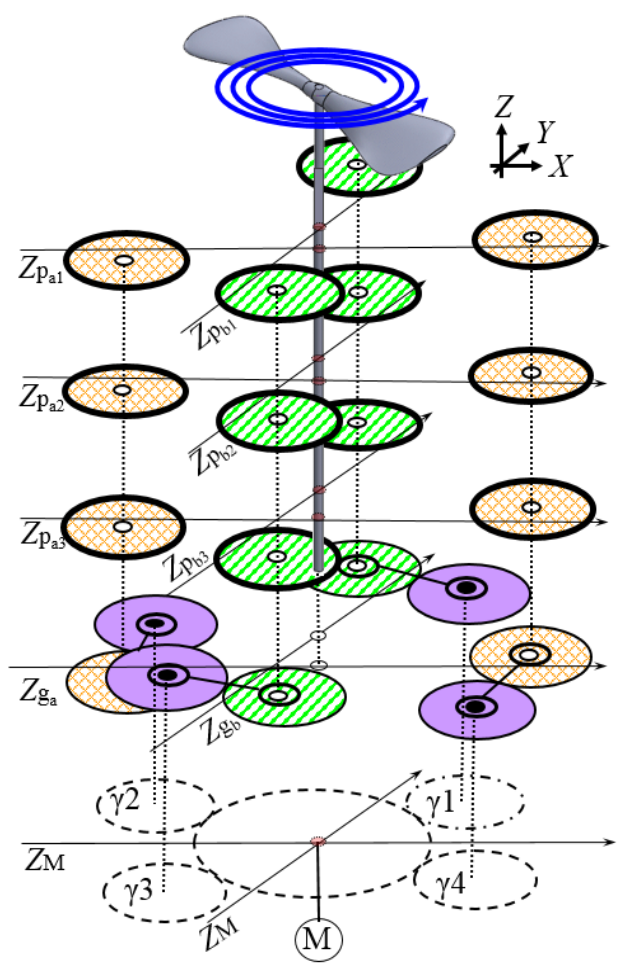

(b) State after release

Fig. 8 Overview of taketombo launching mechanism in Proposal B

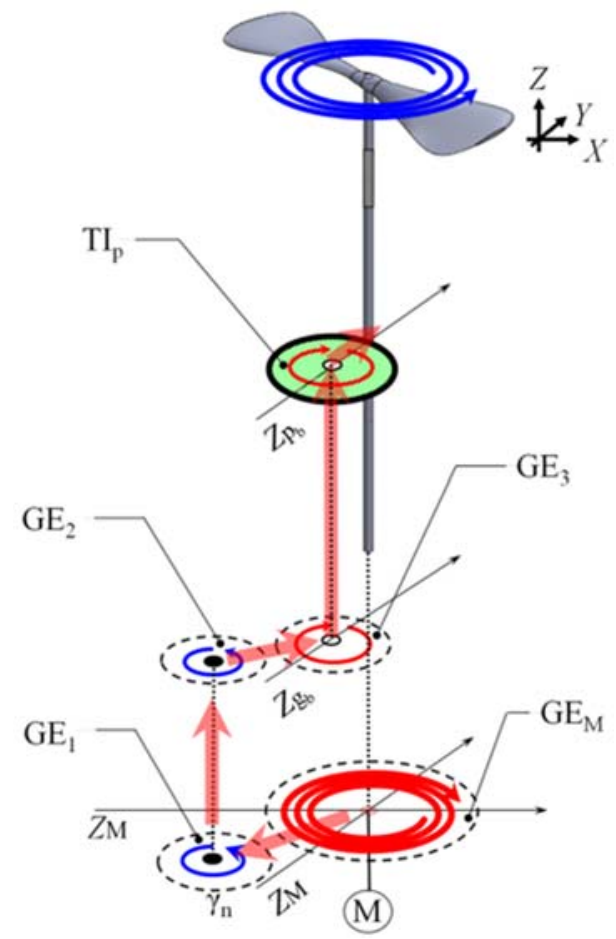

Table 1 Launcher gear specifications

\begin{tabular}{|c|c|c|c|}
\hline Part names & $\begin{array}{c}\text { Parameter } \\
\text { (ratio or } \\
\text { diameter) }\end{array}$ & $\begin{array}{c}\text { Number } \\
\text { of teeth } \\
\text { per gear }\end{array}$ & Value \\
\hline Geared motor unit & $R_{\mathrm{M}}$ & --- & $1 / 20$ \\
\hline $\mathrm{GE}_{\mathrm{M}}$ & $D_{\mathrm{M}}$ & 24 & $36[\mathrm{~mm}]$ \\
\hline $\mathrm{GE}_{1}$ & $D_{1}$ & 30 & $45[\mathrm{~mm}]$ \\
\hline $\mathrm{GE}_{2}$ & $D_{2}$ & 18 & $27[\mathrm{~mm}]$ \\
\hline $\mathrm{GE}_{3}$ & $D_{3}$ & 18 & $27[\mathrm{~mm}]$ \\
\hline $\mathrm{TI}_{\mathrm{p}}$ & $D_{\mathrm{p}}$ & --- & $43[\mathrm{~mm}]$ \\
\hline Taketombo shaft & $d_{\mathrm{b}}$ & --- & $3.5[\mathrm{~mm}]$ \\
\hline
\end{tabular}

Fig. 9 Launching mechanism in Proposal B, focusing on the $\gamma$-axis

A concrete design was produced based on this examination result. The required motor power was estimated with reference to the existing taketombo launcher. In our preliminary design, the output performance required to accelerate a 
taketombo having a moment of inertia $8.3 \times 10^{-5}\left[\mathrm{kgm}^{2}\right]$ to a rotation speed of 3000 [rpm] within 0.3 [s] was set as the target. The rotational energy of $3000[\mathrm{rpm}](=314[\mathrm{rad} / \mathrm{s}])$ was set to about $4[\mathrm{~J}]$. The minimum value necessary if the taketombo is to accelerate from a stopped condition to the target number within 0.3 [s] is 13 [W], so this value was a set as a standard in this study and incorporated into the design.

Since a small motor capable of exceeding 10,000 [rpm] was used, the geared motor was combined with a reduction gear. The reduction unit of the geared motor can be chosen from 1/10 to 1/300. An example combined with the gear is shown in Table 1. In this example, a transmission ratio $N_{\mathrm{M}} / N_{\mathrm{b}}$ is set to about 2 . Additionally, if exchanged for $1 / 10$ in the reduction gear unit $\left(R_{\mathrm{M}}\right)$, the final velocity ratio will be set to about 1 . This ratio is close to a direct motor and taketombo axial shaft connection and allows the launcher to achieve double the running torque easily with half the rotation speed.

\subsection{Example of fabrication}

The generally available and inexpensive FDM 3D printer system was used for the fabrication of this device. While ABS resin was selected for most of the components, easily obtainable commercial parts were used for important functions requiring high stiffness levels, such as the hardening processing axis, ball bearing, and metal collar, and components manufactured by 3D printer were used for connections between these purchased parts.

Furthermore, the 3D print gear, in which wear is a problem, was changed from a spur gear to a helical gear, dealt with partial wear. Moreover, gear parts that were deemed susceptible to strength reduction were redesigned and increased in thickness. The erection diagram created with 3D CAD software is shown in Fig. 10 (a). However, auxiliary parts, such as the servo required for a release mechanism, have been omitted. Fig. 10 (b) shows the fully assembled launcher. The total weight of this launcher is $1.6 \mathrm{~kg}$, and the external size is $12 \mathrm{~cm} \mathrm{x} 12 \mathrm{~cm}$ x $30 \mathrm{~cm}$.

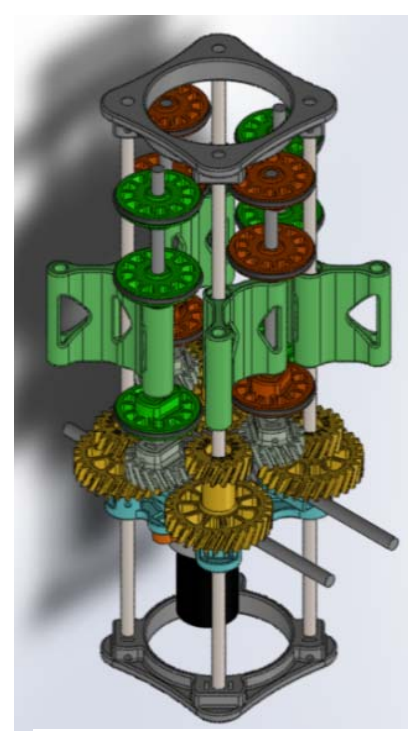

(a) 3D CAD model

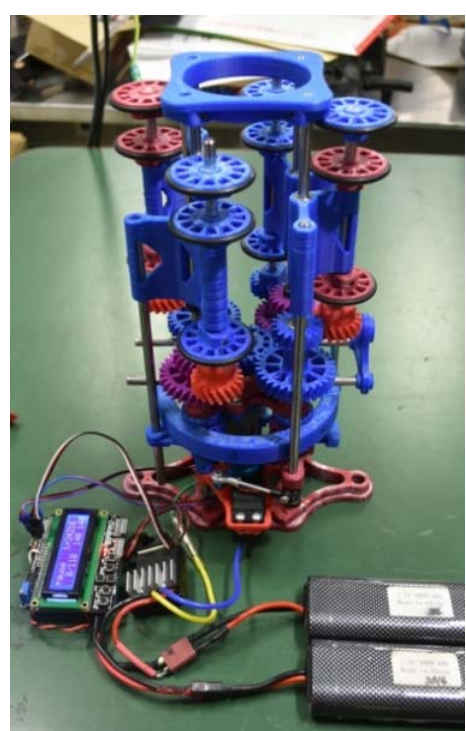

(b) Prototype device using the proposed methodology

Fig. 10 Mechanical launching device

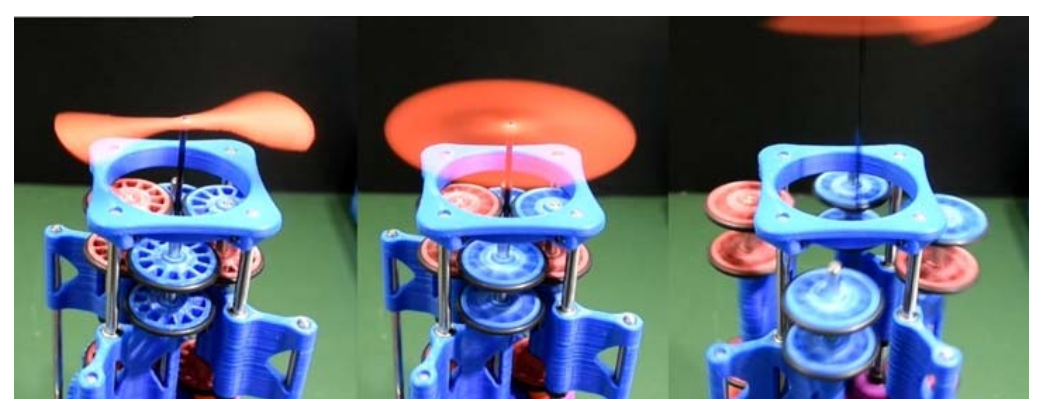

(a) Gripping state

(b) Rotation state

(c) State after release

Fig. 11 Taketombo launching sequence using the prototype device 


\section{Experimental results}

The operating state at the time of a launcher is loaded with a taketombo made of ABS resin is shown in Fig. 11.

Furthermore, in order to confirm launching accuracy, the arrangement plan at the time of ejection is shown in Fig. 12. In this figure, $\mathrm{C} 1$ and $\mathrm{C} 2$ indicate the installation positions of the cameras used to record the experiment.

Fig. 13 consists of sequential photographs from $\mathrm{C} 1$ taken at the time a taketombo is launched, while the sequential photographs from $\mathrm{C} 2$ are shown in Fig. 14. As can be seen in Fig. 13, the taketombo gains altitude immediately after release. In Fig. 14, it can be seen drifting slightly to the upper right as it climbs (the yellow dashed circles show the taketombo position). Finally, as a durability check, approximately 100 consecutive trial launches were conducted in continuous rotation over a period of 2 hours (equivalent to approximately 1000 trial launches), confirming sufficient durability of the device.

We recognize that the reproducibility of the flight path at the same condition is more important than the durability of the device. We tried a few trials and confirmed qualitatively that it may have reproducibility. However, at the present time, the reproducibility cannot be proven since we do not have a flight path measurement system. Our further study is to construct the flight path measurement system and to show the reproducibility of the flight path quantitatively.

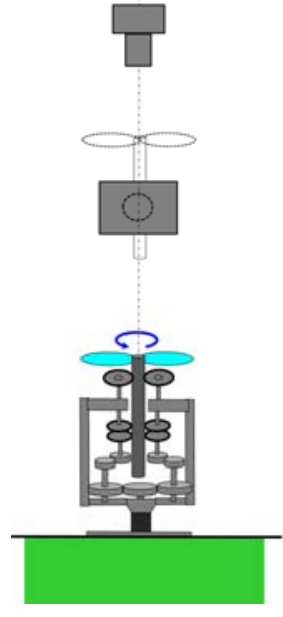

(a) Front View

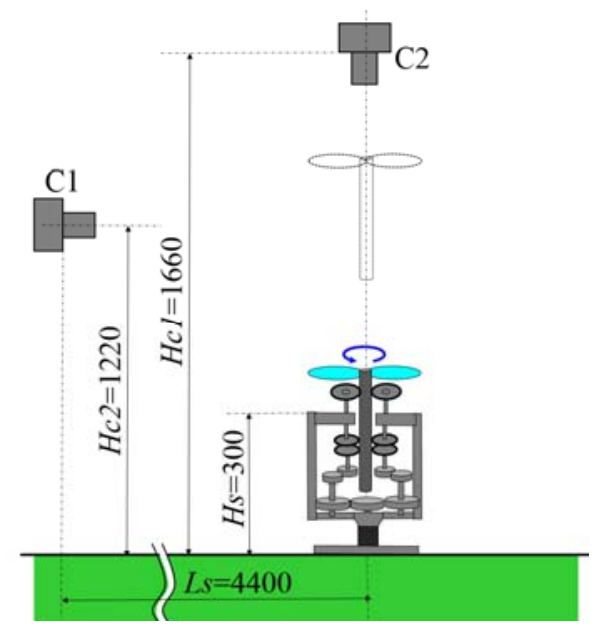

(b) Side View

Fig. 12 Arrangement of experiment devices
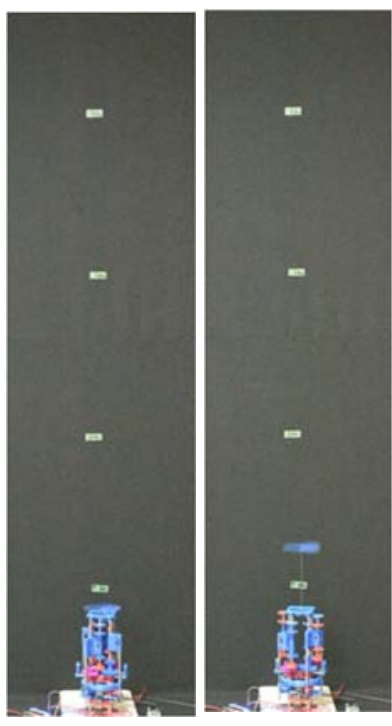

$t=0 / 6[\mathrm{~s}] \quad t=1 / 6[\mathrm{~s}]$

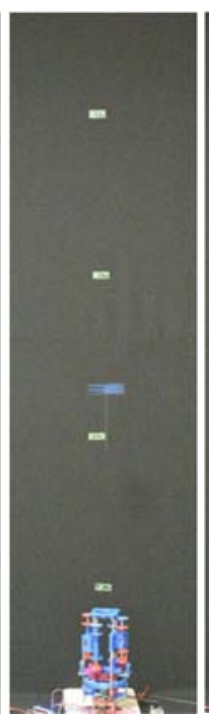

$t=2 / 6[\mathrm{~s}]$

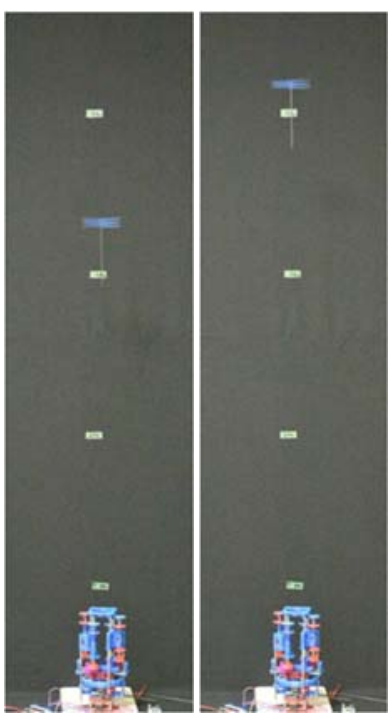

$t=3 / 6[\mathrm{~s}] \quad t=4 / 6[\mathrm{~s}]$

Fig.13 Measurement results from C1 


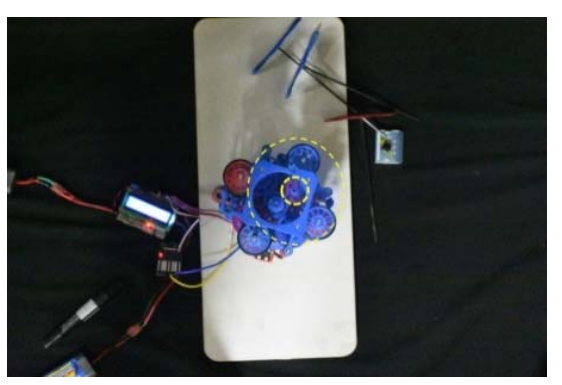

$t=2 / 6[\mathrm{~s}]$

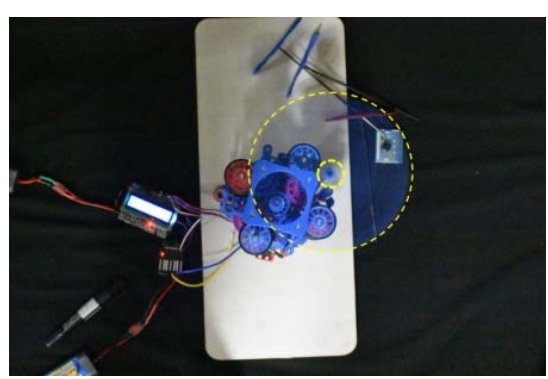

$t=3 / 6[\mathrm{~s}]$

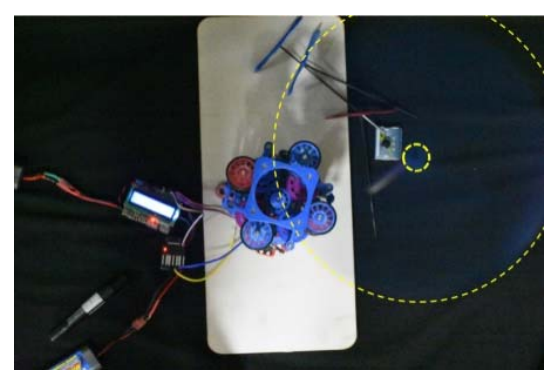

$t=4 / 6[\mathrm{~s}]$

Fig. 14 Measurement results from C2

\section{Conclusion}

In this paper, we discussed the need for equipment that can facilitate drone development, focusing on launchers that could be used to evaluate the aerodynamics of experimental rotary wings. We then reported on a newly developed device that is capable of imitating the actions of a person at the time launching a taketombo. In experiments aimed at imitating the action of a person launching such a device, attention was paid to three mechanisms: axial shaft grasping, axial shaft rotation, and axial shaft release. We began the conceptual design process for our device by determining the best way to imitate each mechanism, after which our focus switched to achieving each mechanism mechanically. Finally a detailed design was produced that took into consideration of the manufacture of component parts fabricated using a 3D FDM printer system.

Once the device was fabricated, taketombo launch experiments were conducted, the results of which suggest that our newly fabricated launcher has the ability to launch a taketombo correctly towards a low-altitude nearby target. Specifically, we performed 100 consecutive trial launches over a period of 2 hours (equivalent to approximately 1000 trial launches) in order to confirm the durability of the device. From these results, the potential utility of the device was confirmed and it was thought that trial production of the launcher could further facilitate aerodynamic ability evaluation of experimental rotary wings.

Our future work will focus on increasing the amount of statistical data obtained from continuous trial launches and investigating the differences in aerodynamic performance from experiments conducted using two or more rotary wings types.

\section{Acknowledgment}

This work was supported by the Japan Society for the Promotion of Science (JSPS) KAKENHI Grant Numbers $17 \mathrm{~K} 01056$ and 16K14500.

\section{References}

Brandt, J.B. and Selig, M.S., Propeller Performance Data at Low Reynolds Numbers, 49th AIAA Aerospace Sciences Meeting, AIAA Paper 2011-1255, (2011).

Chen, F. Y., Gripping Mechanisms for Industrial Robots, Mechanism and Machine Theory, Vol.17, No.5 (1982), pp.299-311.

Keeney, T., Amazon Drones Could Deliver a Package in Under Thirty Minutes for One Dollar, ARK Investment Management, 2015-12-01, available from <https://ark-invest.com/research/amazon-drone-delivery $>$, (accessed on 1 July, 2017).

Lee, J., Kinematic Synthesis of Industrial Robot Hand/Gripper - A Creative Design Approach, Robotics and Autonomous Systems, Vol.4, No.3 (1988), pp.257-263.

Nonami, K., State of the Art Issue of Drone Technology and Business Frontier, Journal of Information Management, Vol. 59, No.11 (2017), pp.755-763 (in Japanese). 
Shetty, O.R. and Selig, M.S., Small-Scale Propellers Operating in the Vortex Ring State, 49th AIAA Aerospace Sciences Meeting, AIAA Paper 2011-1254, (2011).

Takato, K. and Shirayama, S., An Engineering Education Toward Sustainable Society Through Manufacturing a Flying Toy Taketombo, Journal of Advanced Mechanical Design, Systems, and Manufacturing, Vol.6, No.7 (2012), pp.1143-1153.

Takato, K. and Shirayama, S., A Study of an Ejection Type Rotorcraft for Observation, Conference Proceedings of the Japan Society of Mechanical Engineers, No.13-1 (2013), G151025 (in Japanese).

Tsuneya, R. and Tsutsumi, M., Research on the Flying Bamboo Helicopters, Bulletin of Nagasaki Institute of Applied Science, Vol.33 (1992), pp.29-51 (in Japanese).

Yasuda, K., Take-off and Aerodynamic Characteristics of a Taketombo, Journal of the Japan Society for Aeronautical and Space Sciences, Vol.56, No.648 (2008), pp.29-33 (in Japanese).

Yasuda, K., Flight Test of a Taketombo, Journal of the Japan Society for Aeronautical and Space Sciences, Vol.64, No.1 (2016), pp.1-5 (in Japanese). 